\title{
Ion Beam Micromachining of Integrated Optics Components
}

\author{
Hugh L. Garvin, E. Garmire, S. Somekh, H. Stoll, and A. Yariv
}

\begin{abstract}
Thin film integrated optics components such as light guides, modulators, directional couplers, and polarizers demand high quality edge smoothness and high resolution pattern formation in dimensions down to submicrometer size. Fabrication techniques combining holographic and scanning electron beam lithography with ion beam micromachining have produced planar phase gratings with intervals as small as 2800 $\AA$, guiding channel couplers in GaAs, and also wire- grid polarizers for $10.6-\mu \mathrm{m}$ radiation.
\end{abstract}

\section{Introduction}

The recent interest in the field of fiber optics communication and integrated optics has been stimulated by the anticipation of thin-film circuitry in which data processing is performed on optical beams and then sent via optical fibers. This application calls for integrated optics components such as light guides, modulators, directional couplers, and similar devices. ${ }^{1}$ These components demand high quality edge smoothness and high resolution pattern formation in dimensions down to submicrometer sizes.

In this paper we describe the use of holographic as well as conventional photolithographic patterns in photoresist that serve as ion beam micromachining (also referred to as back sputtering or ion beam milling) masks in the fabrication of integrated optics components in single crystalline GaAs. We also report briefly on the use of electron beam lithography combined with ion beam machining to produce planar phase grating couplers on $\mathrm{ZnS}$ optical guides.

\section{Ion Beam Sputtering}

The sputtering process provides a convenient method for thin-film deposition and removal that can be applied to a wide variety of materials. The term sputtering refers to the ejection of atoms from a target surface by bombardment with energetic particles (usually ions with energies in the range of $0.6-20$ $\mathrm{keV})$. The ejected atoms can be either removed from a target substrate or deposited on an opposing substrate. Plasma sputtering is widely used in the microelectronics industry ${ }^{2}$ and has also been used in the fabrication of glass waveguides. ${ }^{3}$ The plasma is

The first author named is with Hughes Research Laboratories, Malibu, California 90265; the other authors are with the California Institute of Technology.

Received 7 September 1972. usually created in an inert gas (at $10^{-1}$ to $10^{-3}$ Torr) by a dc or rf discharge and the sputtering occurs by ion bombardment from the plasma. Ion beam sputtering is performed by bombarding the target surface with a collimated ion beam in high vacuum. This type of sputtering has been proved to be a useful alternative in instances where high vacuum conditions are desired for film deposition or when control and direction of micromachining is important. ${ }^{4}$

For fabrication purposes an inert gas ion beam is produced in a duoplasmatron-type ion source (as shown in Fig. 1), accelerated through a high vacuum chamber, and directed (with or without final focusing) to impinge on a target surface. The high energy (typically 3-10 keV) impact of the ions causes sputtering ejection of the target material. By means of contact masking or by shadow masking, patterns can be machined (milled) into the surface of the target. The directed beam permits etching of patterns that are more sharply defined than those of chemical etching and because no material is impervious to the sputtering attack, high resolution patterns can be produced in a broad variety of thin-film materials.

A broad ion beam bombarding a target is used to accomplish high vacuum deposition of the sputtered target material onto an opposing substrate. This is illustrated by the alternate substrate position shown in Fig. 1. Electrons provided by the neutralizing filament are trapped in the potential of the ion beam and are available for continuous neutralization of any positive surface charge that may tend to accumulate. Therefore, dc bombardment suffices for sputtering deposition or removal of either metallic or insulating materials.

Since the back-sputtering rate proceeds uniformly in a given homogenous material, the three-dimensional shape of a contact mask will be fairly well replicated in the substrate if sputtering continues until the mask is just etched away. Some distortion of the cross-sectional detail occurs because the sputter- 


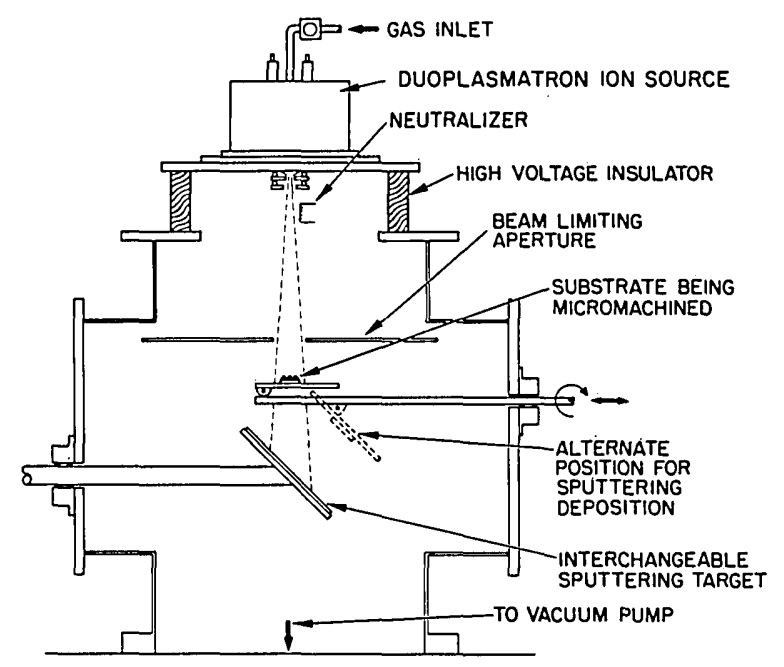

Fig. 1. Schematic diagram of the duoplasmatron ion beam sputtering system.

ing rate varies with the angle of incidence. Also, the depth of etching into the substrate may differ from the depth of the photoresist removed if the sputtering yields of the two materials differ.

\section{Corrugation in Optical Waveguides}

Periodic structures in optical waveguides can be made by corrugation of the waveguide surface. By choosing the right periodicity it is possible to convert power from one mode to another ${ }^{5}$ or couple power between a confined guided mode and an unconfined radiation mode, thus forming a grating coupler. ${ }^{6}$ Mode conversion from a forward propagating mode to a backward propagating mode forms a distributed mirror. This kind of a mirror may help in extending the lifetime of semiconductor injection lasers in the cases where mirror damage is the dominant failure mode. If the corrugation extends along the length of an amplifying medium, a distributed feedback laser results. ${ }^{7}$ In general, corrugating the surface of an optical thin-film waveguide produces space harmonics with phase velocities that depend on the corrugation period. These can be used in interactions that require phase matching, such as in nonlinear optics experiments and devices. 8

The periods of the corrugations needed for these applications vary from as low as approximately 0.13 $\mu \mathrm{m}$ for a distributed feedback laser in GaAs to tens of microns for the phase matching of $10.6-\mu \mathrm{m}$ second harmonic generation in GaAs. We have employed two approaches for fabricating gratings and found them both successful. In the first approach a computer-programmed scanning electron microscope (SEM) was used to write a grating pattern of 0.1 $\mu \mathrm{m}$-wide lines with a period of $0.37 \mu \mathrm{m}$ in a masking material of polymethyl methacrylate (PMM). After dissolving away (developing) the material in the exposed (or written) areas, a broad-area argon ion beam was used to etch the grooves into the substrate (in this case $\mathrm{ZnS}$ ) using the PMM as a sputter mask.
Figure 2 shows the resulting grating in a waveguide of $\mathrm{ZnS} 10 \mu \mathrm{m}$ wide by $2 \mu \mathrm{m}$ thick. The depth of the etching is $500 \AA$.

Using an alternative approach, the grating mask pattern was produced by laser holographic techniques. The surface of the guiding layer was spincoated with a photosensitive resist material (both KPR or Shipley 1350 resists were used; manufacturers Eastman Kodak Company and Shipley Company, Inc.). Argon laser (4580- $\AA$ line) beams from a common source were projected onto the resist at an angle, as shown in Fig. 3(a). The interference of these two beams caused exposure with a sinusoidal variation across the surface at a period of $d=\lambda / 2$ $\sin \alpha$. By adjustment of the exposure and development parameters the pattern is left as a partial coverage on the surface of the substrate [Fig. 3(b)]. Although lower exposure and development leaves the undulating pattern on the surface of the resist layer 9 and, in principle, the material can be ion-machined away leaving a replica of the undulations in the substrate surface, this approach is difficult to control if
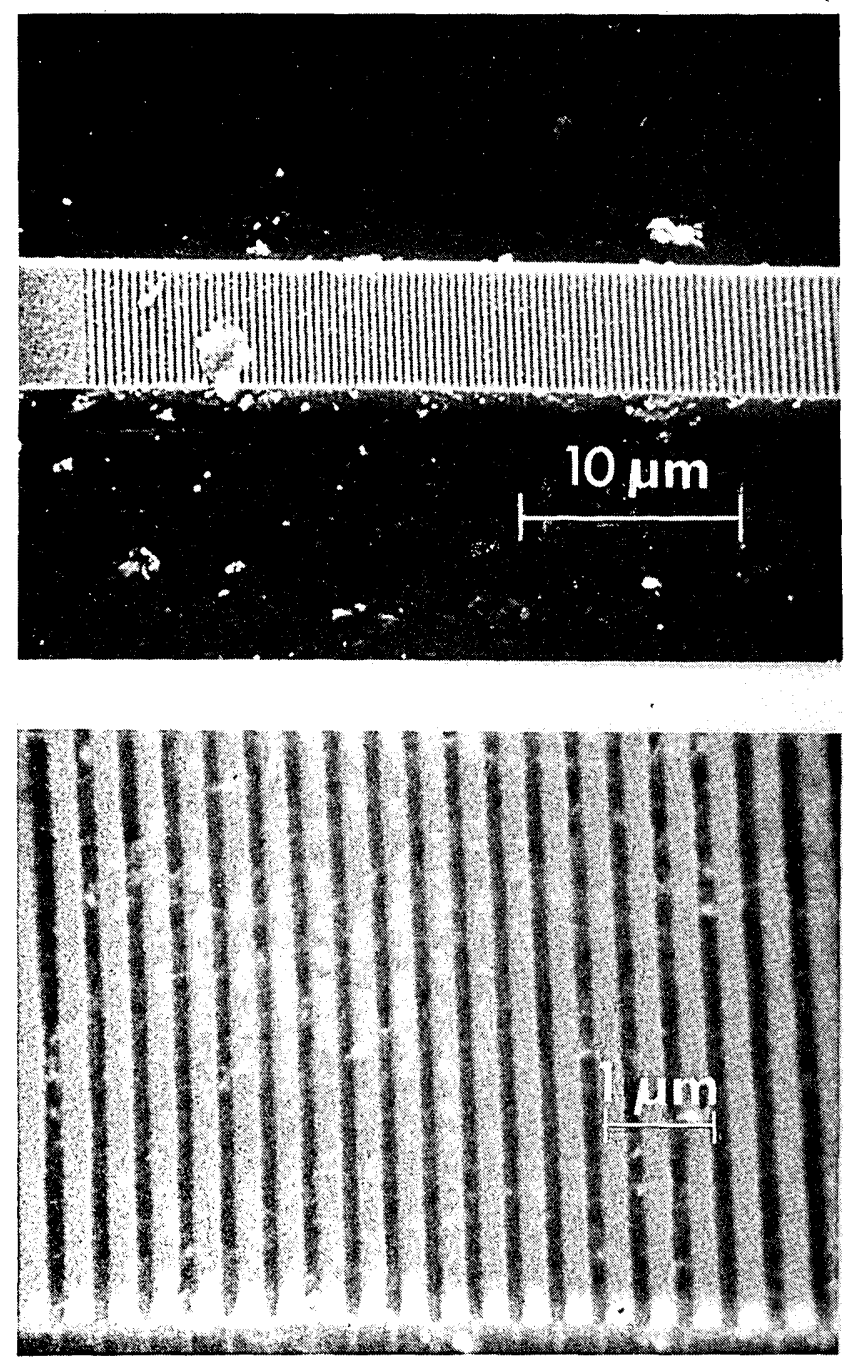

Fig. 2. Planar grating coupler with 1000- $\AA$ grooves ion beam machined into an optical waveguide. Periodicity is $3700 \AA$. 


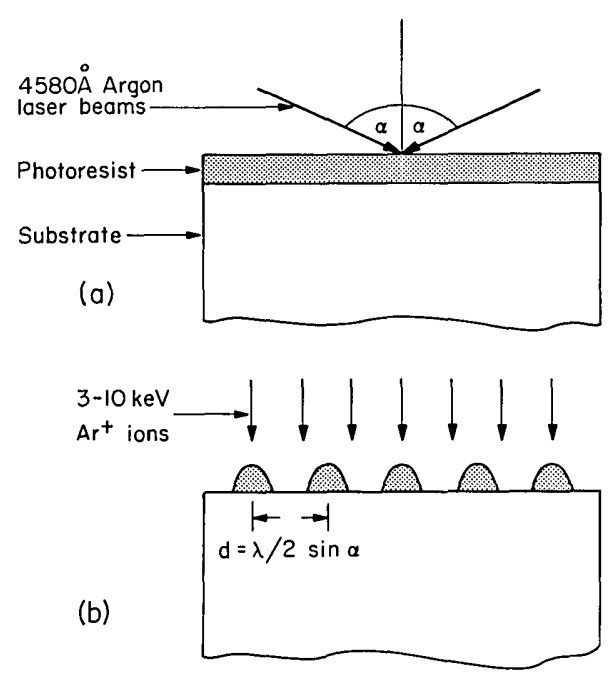

Fig. 3. (a) Holographic exposure of photoresist. (b) Ion machining of photoresist after development.

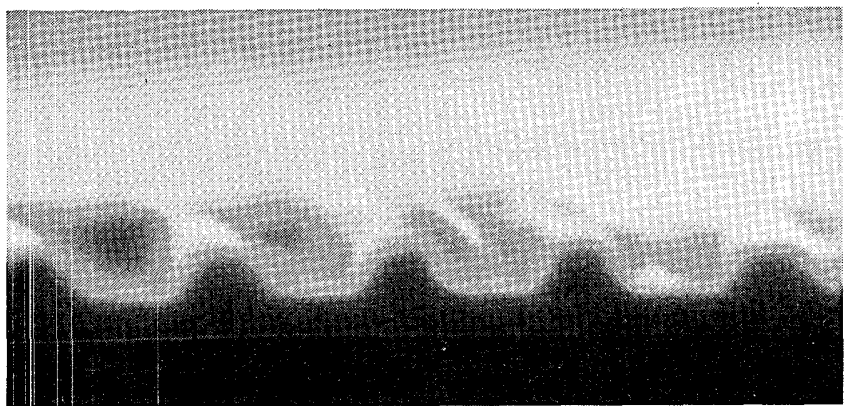

Fig. 4. Cross section of grating pattern $(0.41-\mu \mathrm{m}$ periodicity) in GaAs. Scanning electron microscope magnification is $55,000 \times$.

the resist material contains volatile components. This is because the ion beam heats the resist material and gas evolution causes bubbles and blisters that lift the pattern and stretch it to eventual destruction. It is more desirable to develop the pattern as clearly defined and separated stripes, which provides for local gas evolution without bubble formation.

We have produced grating patterns with periods of $0.28 \mu \mathrm{m}, 0.41 \mu \mathrm{m}, 1.4 \mu \mathrm{m}$, and $3.0 \mu \mathrm{m}$ using holographic techniques. Ion beam machining was then employed to etch these structures into the surface of silicon and GaAs substrates. The $0.41-\mu \mathrm{m}$ pattern was etched to a depth of $0.12 \mu \mathrm{m}$ into the surface of GaAs as can be seen from the SEM cross-section photograph (Fig. 4). Figure 5(a) shows a lower magnification photograph of the 1.4- $\mu \mathrm{m}$ grating, and Fig. 5 (b) shows the smallest period grating that we have machined, $0.28 \mu \mathrm{m}$. The smallest period was determined by the laser wavelength rather than the pho-
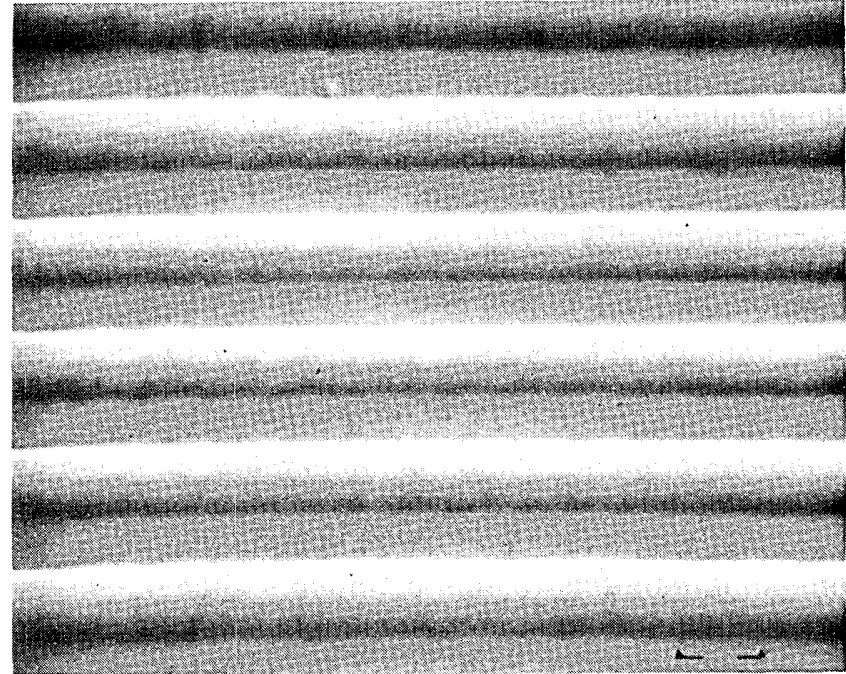

(a)

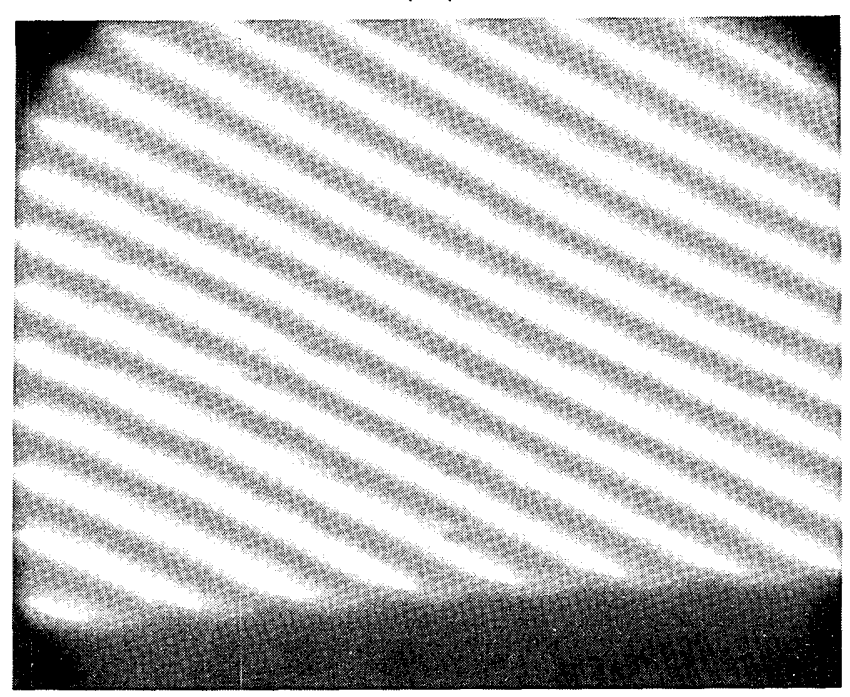

(b)

Fig. 5. SEM photographs of (a) 1.4- $\mu \mathrm{m}$ grating in GaAs, and (b) $0.28-\mu \mathrm{m}$ grating in $\mathrm{Si}$.

toresist resolution, which means that smaller period gratings can be fabricated using a shorter wavelength light. We believe that the high resolution capabilities of the SEM ${ }^{10}$ would also enable us to fabricate gratings with periods down to $0.13 \mu \mathrm{m}$, which are needed for distributed feedback lasers in GaAs.

\section{Optical Circuitry}

Ion machining combined with either conventional photolithography or scanning electron lithography is very useful in the fabrication of optical circuitry. Components such as straight and curved-channel waveguides, directional couplers, ${ }^{11}$ thin-film analogs of lenses and prisms, ${ }^{12}$ etc. can be constructed by this technique. The ion beam can be used to copy structures in the exposed photoresist (or electron resist) into the guiding material or into a specific masking material that is used whenever the guides are made by ion implantation ${ }^{13,14}$ or diffusion. ${ }^{15}$ 
As an example, we report here the fabrication of gold masks for proton-implanted waveguides in GaAs. Bombardment of $\mathrm{GaAs}$ with $300-\mathrm{keV}$ protons has been shown to form 3 - $\mu \mathrm{m}$-thick waveguides. ${ }^{13}$ Since a gold layer $1.5 \mu \mathrm{m}$ thick can completely block these protons, an optical circuit can be implanted if a suitable gold mask is constructed on the surface of the GaAs substrate.

In the fabrication of a large number of channel waveguides closely spaced so as to allow directional coupling between them we have used a conventional photographic mask. A GaAs substrate was sputtercoated with a gold layer $1.8 \mu \mathrm{m}$ thick. Finally, a photoresist layer $(2 \mu \mathrm{m})$ was spin-deposited and was exposed through a mask of transparent stripes. The exposed resist was developed down to the gold to form stripes of resist with a ridge shape. The succeeding step was ion machining of the clear areas of gold down to the substrate. Figure 6(a) and (b) show the resulting gold stripes with the remainder of the photoresist on top of them. The periodicity of the structure is about $6.4 \mu \mathrm{m}$. In the clear areas between the gold stripes the proton implantation takes place and the channel waveguides are formed. The optical measurements of these guides and the coupling between them is the subject of a forthcoming paper. ${ }^{16}$

As can be seen in Fig. 6(b), the side walls of the gold stripes are quite rough. The reason is the limited quality of the photographic mask. A better wall definition can be achieved by using one of the methods described earlier, namely, scanning electron lithography or the holographic technique. (Note the wall's sharpness in Fig. 5.) In some cases a photographic mask can be replaced by a better-quality mask that is produced by diamond scribing through a metal film on a glass slide. Figure 7 shows a mask prepared for the fabrication of straight and curved $10-\mu \mathrm{m}$ wide waveguides. With this mask pattern the directionality of ion beam machining produces smooth, straight sides in the masking or guiding layer (see Fig. 2) with no undercutting or ragged edges.

The pattern of fine gold lines, as described above, has another application in the electrooptical field, namely, as a wire-grid polarizer for infrared radiation. ${ }^{17}$ In this application the gold film is deposited onto a substrate such as Irtran 2 (Eastman Kodak Co. trademark), CdTe, or germanium, which is transparent to the desired radiation (e.g., $10.6-\mu \mathrm{m}$ laser radiation). The photoresist mask is applied by the techniques described above, and ion beam machining is used to etch through the exposed gold down to the substrate material. This method of etching is particularly desirable when the surface of the substrate is coated with films of antireflecting materials that would be damaged by chemical etching processes. In preliminary studies of polarizers produced by this technique an extinction ratio of greater than $99 \%$ has been demonstrated by a 1.1 $\mu \mathrm{m}$ grating interval, and further improvement is expected with refinements in the pattern design.

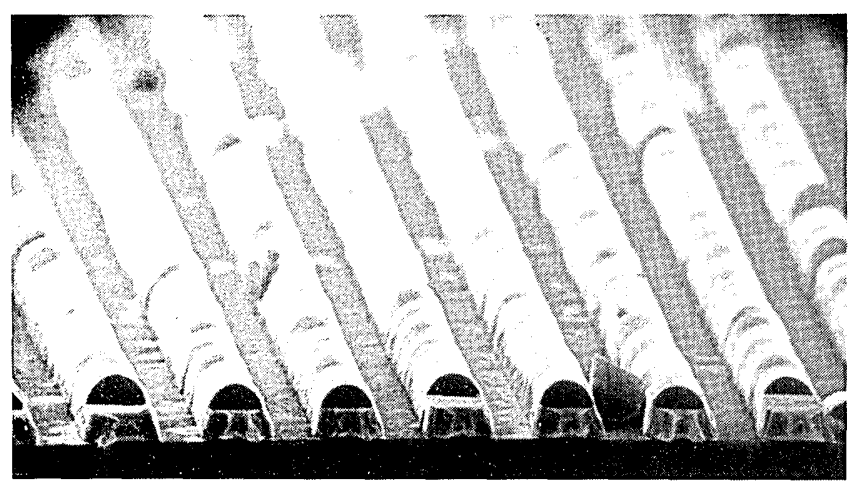

(a)

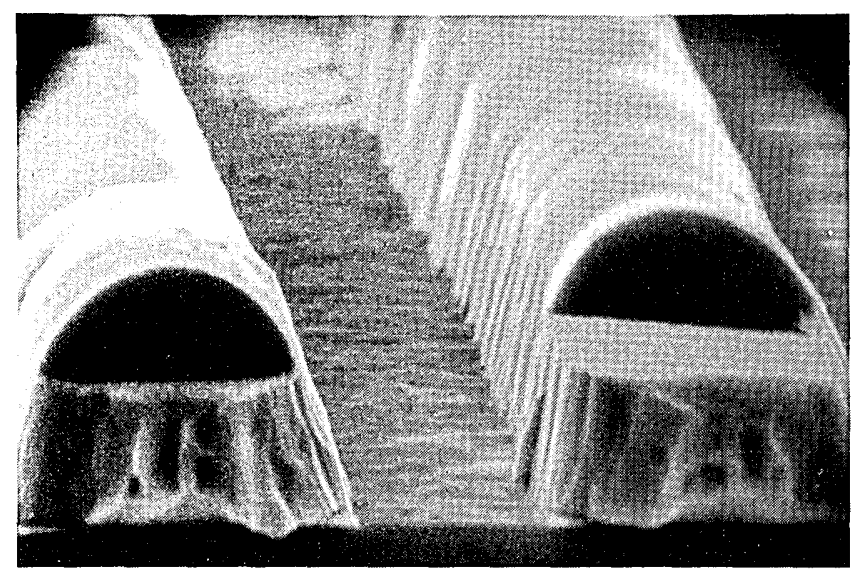

(b)

Fig. 6. SEM photographs of the gold mask on the GaAs substrate, used in the fabrication of the directional couplers. The remains of the photoresist that defines the channels when the gold mask is machined can be seen on top of the gold stripes. (a) Magnification $2100 \times$, (b) magnification $10,400 \times$.

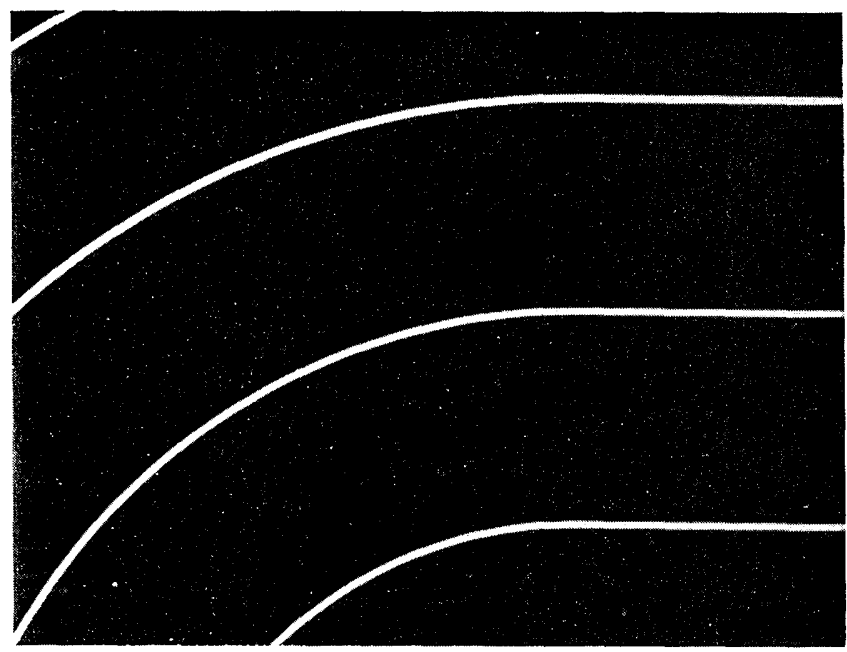

Fig. 7. Scribed metal photomask of optical guide pattern. Guide width is $10 \mu \mathrm{m}$.

\section{Conclusions}

Ion beam machining has been found to be a very useful tool in the fabrication of integrated optics components. When it is combined with the high resolution lithography of a scanning electron micro- 
scope, the fabrication of components with submicron elements becomes possible.

For the fabrication of grating-type corrugations at various periodicities the combination of holographic exposure of photoresist and ion beam machining has been proved to be a controllable and reproducible process. When conventional photolithography is used together with ion beam machining, the quality of the pattern definition and hence the performance of the resultant electrooptical device appears to be limited only by the quality of the photographic mask and the optical replication process.

The authors wish to express their gratitude to J. R. Devaney and K. C. Evans at the Jet Propulsion Laboratory for the scanning electron microscope photographs shown in Figs. 4, 5(b), and 6(a) and (b). We also wish to express our appreciation to E. D. Wolf and W. E. Perkins of the Hughes Research Laboratories for the electron lithography work leading to Fig. 2, and to P. J. Coane for the scanning electron micrograph shown in Fig. 5(a).

\section{References}

1. A. Yariv, "Active Integrated Optics," in Proceedings, 1971 Esfahan Conference on Pure and Applied Laser Physics (Wiley, New York), to be published.

2. Many applications have been cited at the Symposia on the Deposition of Thin Films by Sputtering, sponsored by the University of Rochester and Bendix Corporation, Rochester,
New York, held in 1966, 1967, and 1969, and at the Conference and School Sessions on the elements, techniques, and applications of sputtering sponsored by the Materials Research Corp., Orangeburg, New York.

3. J. E. Groell and R. D. Standley, Bell Syst. Tech. J. 48, 3445 (1969).

4. H. L. Garvin, in Proceedings, 3rd Symposium on the Deposition of Thin Films by Sputtering (Bendix Corp., Rochester, N.Y., 1969), pp. 4-11.

5. D. Marcuse, Bell Syst. Tech. J. 48, 3177 (1969).

6. M. L. Dakss, L. Kuhn, P. F. Heidrich, and B. A. Scott, Appl. Phys. Lett. 16 (12), 523 (1970).

7. H. Kogelnik and C. V. Shank, Appl. Phys. Lett. 18, 152 (1971).

8. S. Somekh and A. Yariv, Appl. Phys. Lett. 21, 140 (1972).

9. R. G. Brandes and R. K. Curran, Appl. Opt. 10, 2101 (1971).

10. E. D. Wolf, F. S. Ozdemir, W. E. Perkins, and P. J. Coane "Response of Elvacite 2041," in Proc. 11th Symposium on Electron, Ion and Laser Beam Technology, R. F. M. Thorley, Ed. (San Francisco Press, San Francisco, 1970), pp. 331-336.

11. E. A. J. Marcatili, Bell Syst. Tech. J. 48, 2071 (1969).

12. R. Shubert and J. H. Harris, J. Opt. Soc. Am. 61, 154 (1971). Also R. Ulrich and R. J. Martin, Appl. Opt. 10, 2077 (1971).

13. E. Garmire, H. Stoll, A. Yariv, and R. G. Hunsperger, Appl. Phys. Lett. 21, 87 (1972).

14. R. D. Standley, W. M. Gibson, and J. W. Rodgers, Appl. Opt. 11, 1313 (1972).

15. H. F. Taylor, W. E. Martin, D. B. Hall, and V. N. Smiley, Appl. Phys. Lett. 21, 95 (1972).

16. S. Somekh, E. Garmire, A. Yariv, H. L. Garvin, and R. G. Hunsperger, Appl. Phys. Lett. 22, 46 (1973).

17. J. P. Auton, Appl. Opt. 6, 1023 (1967).

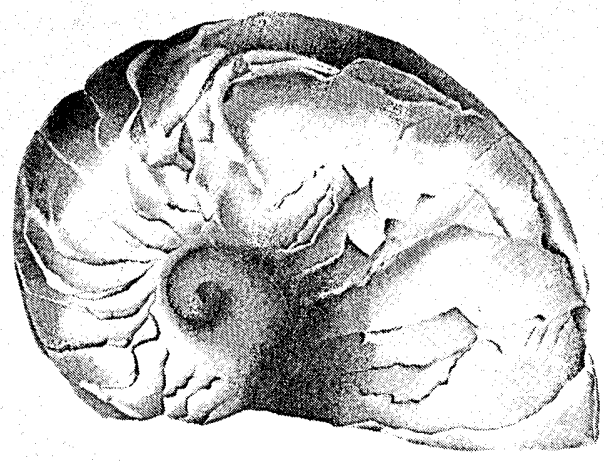

